\title{
Coherent CFAR detection in compound Gaussian clutter with inverse gamma texture
}

\author{
Graham V Weinberg
}

\begin{abstract}
Recent publications have explored coherent radar detection in a compound Gaussian clutter environment with inverse gamma texture, since the latter clutter model has been validated for $\mathrm{X}$-band high-resolution maritime surveillance radar clutter returns. This paper explores the development of coherent constant false alarm rate (CFAR) detectors for this scenario. In the first instance, a detector is constructed with explicit knowledge of the clutter parameters. It is then shown that the probability of false alarm/threshold relationship does not vary with the clutter power. To achieve a CFAR detector, clutter parameter approximations are then introduced, and the cost associated with this is then analysed.
\end{abstract}

Keywords: Coherent detection, CFAR, Inverse gamma texture, Pareto intensity clutter models, Neyman-Pearson detection

\section{Introduction}

The compound Gaussian clutter model has provided radar researchers and engineers with a mathematically tractable model for correlated non-Gaussian radar returns $[1,2]$. This clutter model has been validated by considerations of observed real radar returns [3]. Essentially, using the theory of spherically invariant random processes (SIRPs), the clutter is modelled as locally Gaussian with a random power level. From the random variable perspective, the clutter vector is a product of a fast fluctuating component (the speckle) and a slowly modulating component (the texture). The speckle is taken as a Gaussian process, while the texture can be selected to generate a specific marginal intensity distribution [4].

Coherent multilook radar detection in heavy-tailed compound Gaussian clutter is a topic of much interest in current signal processing research [2,5-11]. Specifically, with the validation of compound Gaussian clutter with inverse gamma texture as a model for $\mathrm{X}$-band highresolution maritime surveillance clutter returns, many publications have been examining appropriate detection schemes. Validation of the resultant Pareto clutter intensity model for X-band radar returns has been included in [12-14]. The analysis of relevant detection schemes

\footnotetext{
Correspondence: Graham.Weinberg@dsto.defence.gov.au Electronic Warfare and Radar Division, Defence Science and Technology Organisation, P.O. Box 1500, Edinburgh, South Australia 5111, Australia
}

can be found in [2,15-18]. Much work has been devoted to examining the Neyman-Pearson optimal detector [19] and its suboptimal variants, such as the generalised likelihood ratio test (GLRT) detector [2,18]. In addition, the Gaussian optimal detector or whitening matched filter (WMF) has been shown to be a useful suboptimal detector for the compound Gaussian clutter model of interest [18]. This is mainly for the case where the clutter is approximately Gaussian distributed, which tended to occur for the vertically polarised case.

Constant false alarm rate (CFAR) detectors are important because they eliminate the sensitivity of the probability of false alarm (Pfa) and threshold relationship to variations in the clutter power level $[20,21]$. Coherent multilook detectors are said to have the CFAR property if their Pfa/threshold relationship is independent of clutter parameters. Recently, a coherent CFAR detector has been examined in [18]. In there, it is shown that a scaled version of the WMF can result in CFAR control with respect to the underlying Pareto clutter parameters. However, this detector was found to have limited application due to the fact that it required a significantly large number of looks to avoid detector saturation [18]. Consequently, it became of interest to investigate whether other such CFAR detectors could be produced.

This paper introduces a new coherent detector for target detection in the clutter model of interest, which has 
explicit dependence on the clutter parameters. It will be shown that this detector's probability of false alarm and threshold relationship does not vary with the clutter power level. To achieve a CFAR detector, approximations are made to these clutter parameters, together with a mismatched threshold. The cost of this is then analysed, and conditions are established under which the resultant false alarm probability is not increased to an undesirable level in practice.

Throughout, detection in compound Gaussian clutter with inverse gamma texture will be also referred to as Pareto coherent detection for brevity. The paper is arranged as follows. Section 2 sets the framework for the analysis to follow. A general detector is then introduced in Section 3, while a CFAR suboptimal version is proposed in Section 4. The latter includes a full analysis of the performance of this suboptimal CFAR from a mathematical perspective. Section 5 examines the performance of these detectors through detector performance curves. These will be based upon simulated clutter, generated with Pareto parameters estimated from real data sets. A simple Gaussian target model will also be used in all performance analysis, as in $[2,18]$. Further assumptions employed will be introduced as appropriate.

\section{Detection framework}

The following framework has been taken from [18], which has been based upon that in [9]. The radar return is $z$, a complex $N \times 1$ vector, and the coherent multilook detection problem is stated in the form

$$
H_{0}: \boldsymbol{z}=\boldsymbol{c} \text { against } H_{1}: \boldsymbol{z}=R \boldsymbol{p}+\boldsymbol{c},
$$

where all complex vectors are $N \times 1$. The statistical hypothesis $H_{0}$ is that the return is pure clutter, while $H_{1}$ is the alternative hypothesis that the return is a mixture of signal and clutter. Vector $\boldsymbol{c}$ is the pure clutter return, and $\boldsymbol{p}$ is the Doppler steering vector, whose components are given by $\boldsymbol{p}(j)=e^{j 2 \pi i f_{D}}$, for $j \in\{1,2, \ldots, N\}$, where $f_{D}$ is the target normalised Doppler frequency (product of target Doppler frequency and radar pulse repetition interval), with $-0.5 \leq f_{D} \leq 0.5$. It is assumed that this is fully known. The complex random variable $R$ is the target model, and $|R|$ is its amplitude.

The Neyman-Pearson Lemma [19] states that the form of the best test of (1) is a likelihood ratio under each of the respective hypotheses, compared to a threshold $\tau$. Hence, if $f_{0}(\boldsymbol{z})$ is the density under $H_{0}$ and $f_{1}(\boldsymbol{z})$ is the density under $H_{1}$, the statistical test that maximises the probability of detection for a fixed probability of false alarm is given by

$$
L(\boldsymbol{z})=\frac{f_{1}(\boldsymbol{z})}{f_{0}(\boldsymbol{z})} \underset{H_{0}}{\stackrel{H_{1}}{\gtrless}} \tau,
$$

where $L(\boldsymbol{z})$ is the likelihood function. The notation $L(z) \stackrel{H_{1}}{\gtrless} \tau$ used in (2) means that we reject $H_{0}$ if and only if $H_{0}$

$L(\boldsymbol{z})>\tau$.

The compound Gaussian clutter is modelled as a stationary stochastic process $c=S \mathcal{G}$, where $\mathcal{G}$ is a zero-mean Gaussian process with covariance matrix $\Sigma$. Throughout, as in $[2,18]$, it will be assumed that this is completely known. Further work in this area will attempt to remove this restriction. It will also be assumed that its inverse is semi-definite positive so that a whitening approach can be applied to the detection problem. This is a valid exercise because a SIRP is unaffected by linear transformations [4]. Hence, we suppose that a Cholesky factorisation exists for $\Sigma^{-1}$ so that there exists a matrix $A$ such that $\Sigma^{-1}=A^{H} A$.

The random variable $S$ in the definition of the compound Gaussian model is non-negative and univariate and determines the form of the resultant marginal intensity distribution of the clutter [4]. For the case of inverse gamma texture, $S$ has density given by

$$
f_{S}(s)=\frac{2 \beta^{\alpha}}{\Gamma(\alpha)} s^{-2 \alpha-1} e^{-\beta s^{-2}},
$$

where $\alpha$ and $\beta$ are non-negative texture distributional parameters. Using the theory of SIRPs, it can be shown that the corresponding clutter intensity model is Pareto, with shape parameter $\alpha$ and scale parameter $\beta$ [16].

We now specify the whitened version of the statistical test (1). By applying the Cholesky factor matrix $A$ to the original statistical test and defining $\boldsymbol{r}=A \boldsymbol{z}, \boldsymbol{n}=A \boldsymbol{c}$ and $\boldsymbol{u}=A \boldsymbol{p}$, we arrive at the statistically equivalent form

$$
H_{0}: \boldsymbol{r}=\boldsymbol{n} \text { against } H_{1}: \boldsymbol{r}=R \boldsymbol{u}+\boldsymbol{n} \text {. }
$$

The transformed clutter process is $\boldsymbol{n}=S A \mathcal{G}$, and $A \mathcal{G}$ is a multidimensional complex Gaussian process, with zero mean but covariance matrix, the $N \times N$ identity matrix [11]. The whitening approach used here is invaluable in reducing the form of the likelihood densities, which simplifies the resultant detector considerably.

Using the formulation above and by applying SIRP theory to construct appropriate densities, the GLRT is shown in $[2,16,18]$ to take the form

$$
L(\boldsymbol{r})=\frac{W(\boldsymbol{r})}{\|\boldsymbol{u}\|^{2}\left(\|\boldsymbol{r}\|^{2}+\beta\right)} \underset{H_{0}}{\stackrel{H_{1}}{\gtrless}} \tau,
$$

where

$$
W(\boldsymbol{r})=\left|\boldsymbol{u}^{H} \boldsymbol{r}\right|^{2}
$$

is the WMF. Detector (5) is known as the GLRT linear threshold detector (LTD) in [2], which has been shown to perform very well in spiky clutter. Throughout, detector 
(5) will be referred to as the LTD for brevity. Here, it is assumed that the clutter scale parameter $\beta$ is completely known. Both these detectors are analysed in [18], where closed form expressions between the Pfa and threshold for each are derived. It is also shown explicitly that both (5) and (6) are not coherent CFAR processes.

However, by scaling the WMF, it is shown in [18] that the detector

$$
T_{1}(\boldsymbol{r})=\frac{W(\boldsymbol{r})}{\|\boldsymbol{u}\|^{2}\|\boldsymbol{r}\|^{2}} \underset{H_{0}}{\stackrel{H_{1}}{\gtrless}} \tau_{1}
$$

operating in Pareto clutter has threshold $\tau_{1}$ given by

$$
\tau_{1}=\mathrm{Pfa}^{-1 / N}-1
$$

This is often referred to as the normalised matched filter (NMF) and has been investigated in $[3,6]$. Its relationship to the optimal Neyman-Pearson detector has been explored in [22]. Consequently, (7) is a coherent CFAR process. Analysis of this detector in [18] showed that it had a tendency to saturate if the number of looks is not sufficiently large. As an example, for a false alarm probability of $10^{-6}$, one would require $N>>30$ looks. The motivation for the work presented here is to examine whether other coherent CFAR processes exist in the context of interest. In particular, it will be of interest to examine, as in the approach of [18], whether transformations of the NMF can yield new detectors with improved performance.

\section{General detector}

This section assumes that the clutter parameters $\alpha$ and $\beta$ are known. In the next section, a CFAR detector will be proposed, based upon approximating these. The main purpose of this section is to propose a new detector, whose $\mathrm{Pfa}$ /threshold relationship does not vary with the clutter power. This will then provide one with a threshold relationship that can be used with the new detector, when approximations of clutter parameters are applied.

The literature contains many examples of CFAR detectors produced from ones not possessing the CFAR property $[8,23,24]$. As an example, the LTD detector (5) has been constructed with the assumption that $\beta$ is known. To produce a suboptimal version, with the CFAR property, a maximum likelihood estimate of $\beta$ could be applied to produce a detector with no dependence on $\beta$. If the $\mathrm{Pfa} /$ threshold relationship also contains clutter parameter dependence, approximations can be introduced to minimise the effects of clutter power fluctuations. Alternatively, a mismatched threshold can be applied to eliminate clutter parameter dependence.

The development of the following detector resulted from an analysis of the LTD (5) and NMF (7) and its dependency on the underlying clutter parameters. Suppose $\kappa>0$ is a known fixed constant, which is independent of both Pareto clutter parameters. Consider the coherent detector given by

$$
T_{2}(\boldsymbol{r})=\frac{W(\boldsymbol{r})-\beta\left(\kappa^{1 / \alpha}-1\right)\|\boldsymbol{u}\|^{2}}{\|\boldsymbol{u}\|^{2}\|\boldsymbol{r}\|^{2}},
$$

with detection threshold $\tau_{2}$. The following result shows that the Pfa and threshold do not vary with the clutter parameters:

Lemma 3.1. The detector (9) has false alarm probability given by

$$
\mathrm{Pfa}=\left(1+\tau_{2}\right)^{-N} / \kappa .
$$

Observe that the choice of $\kappa=1$ results in the CFAR detector (7), and so is a special case of (9).

The proof of Lemma 3.1 is now outlined and follows closely the proof of analogous results in [18]. Throughout, $\mathrm{P}$ denotes probability, while $\mathrm{E}$ is the statistical mean with respect to P. By conditioning on $S$ and then $\|\boldsymbol{r}\|^{2} \mid\{S=s\}$, it can be shown that

$$
\begin{aligned}
\mathrm{Pfa}= & \int_{0}^{\infty} \int_{0}^{\infty} f_{S}(s) f_{\|\boldsymbol{r}\|^{2} \mid\{S=s\}} \times \\
& \mathrm{P}\left(W(\boldsymbol{r})>\left(\beta\left(\kappa^{1 / \alpha}+\tau t\right)\|\boldsymbol{u}\|^{2} \mid\{S=s\}, H_{0}\right)\right) d t d s,
\end{aligned}
$$

where $f_{S}$ is the density (3) and $f_{\|\boldsymbol{r}\|^{2} \mid\{S=s\}}$ is the density of $\|\boldsymbol{r}\|^{2}$ conditioned on the event $\{S=s\}$. Using the fact that under $H_{0},\|\boldsymbol{r}\|^{2} \mid\{S=s\}$ has a gamma distribution with parameters $N$ and $s^{-2}$ while $W(\boldsymbol{r}) \mid\{S=s\}$ has an exponential distribution with parameter $s^{-2}\|\boldsymbol{u}\|^{-2}$ (see [18]), it can be shown that the integrals in (11) simplify to

$$
\begin{aligned}
\mathrm{Pfa} & =\frac{2 \beta^{\alpha}}{\Gamma(\alpha) \Gamma(N)} \int_{0}^{\infty} s^{-2 \alpha-2 N-1} e^{-s^{-2} \beta \kappa^{1 / \alpha}} \\
& \int_{0}^{\infty} t^{N-1} e^{-t s^{-2}(1+\tau)} d t d s \\
& =\frac{2 \beta^{\alpha}}{\Gamma(\alpha)}(1+\tau)^{-N} \int_{0}^{\infty} s^{-2 \alpha-1} e^{-\beta \kappa^{1 / \alpha} s^{-2}} d s \\
& =\frac{\beta^{\alpha}}{\Gamma(\alpha)}(1+\tau)^{-N} \int_{0}^{\infty} x^{\alpha-1} e^{-\beta \kappa^{1 / \alpha} x} d x \\
& =(1+\tau)^{-N} / \kappa,
\end{aligned}
$$

where the definition of the gamma function has been used to simplify the integral with respect to $t$, and the transformation $x=s^{-2}$ has then been applied, together 
with a second application of the definition of the gamma function. This completes the proof.

The factor $\kappa$ can be used to minimise the effects of detector saturation, as observed in the practical implementation of the CFAR detector (7). For a small false alarm probability, the threshold set by (8) can be quite large if the number of looks is smaller than around 30 [18]. Such a number of looks will only be achieved if the radar's scan rate is slow. Hence, the factor $\kappa$ should be able to mitigate the effects of having only a small number of looks.

It is now important to determine suitable choices for $\kappa$. Since the threshold is given by

$$
\tau_{2}=(\kappa \mathrm{Pfa})^{-1 / N}-1,
$$

it is necessary to ensure that this threshold is nonnegative. Hence, in view of (13), we must constrain $(\kappa \mathrm{Pfa})^{1 / N}<1$. Consequently, suppose we select a $\kappa$ such that $\kappa \mathrm{Pfa}=10^{n}$, for some $n$. Then, it is immediate that we must choose $n<0$ to ensure the threshold is non-negative.

In addition, since the detector (7) has a tendency to saturate because of large thresholds, we can, without loss of generality, aim to choose a $\kappa$ that results in $\tau_{2}<1$. This requires $(\kappa \mathrm{Pfa})^{-1 / N}<2$, which can be shown to require $n>-N \frac{\log (2)}{\log (10)} \approx-0.3010 N$.

Again, without loss of generality, constrain $\kappa>1$. This assumption will be useful in the analysis to follow. If we assume that the false alarm probability is of the form $\mathrm{Pfa}=$ $10^{-m}$, for some $m>0$, then $\kappa=10^{m+n}>1$ provided $n>-m$. Hence, we can choose any $n$ such that

$$
\max \{-m,-0.3010 N\}<n<0 \text {. }
$$

Further insight into the selection of an appropriate $n$ can be gained by examining the limiting behaviour of the detection probability of (9) as $n \rightarrow 0$. Note that, with the choice of $\kappa$ above, we can write the detection threshold explicitly as a function of $n$ as $\tau_{2}(n)=10^{-n / N}-1$, which converges to zero as $n \rightarrow 0$. Notice also that $\kappa=\kappa(n)$ converges to $10^{m}=\mathrm{Pfa}^{-1}$ as $n \rightarrow 0$. Hence, writing the probability of detection ( $\mathrm{Pd}$ ) as a function of $n$, it is clear with an application of (9) that

$$
\begin{aligned}
\operatorname{Pd}(n)= & \mathrm{P}\left(W(\boldsymbol{r})>\beta\left(\kappa(n)^{1 / \alpha}-1\right)\|\boldsymbol{u}\|^{2}\right. \\
& \left.+\tau_{2}(n)\|\boldsymbol{u}\|^{2}\|\boldsymbol{r}\|^{2} \mid H_{1}\right) .
\end{aligned}
$$

In order to examine the limiting behaviour of (15) as $n \rightarrow 0$, we require an application of functional analysis limit theorems to justify taking the limit inside the probability in (15). The relevant result is Lebesgue's dominated convergence theorem $[25,26]$. Here, we apply it with $n \rightarrow 0$, instead of an infinite limit; it is not difficult to recast the theorem for the case under consideration. Define a sequence of indicator random variables with
$X_{n}\left(\boldsymbol{r}=\mathbf{I}\left[W(\boldsymbol{r})>\beta\left(\kappa(n)^{1 / \alpha}-1\right)\|\boldsymbol{u}\|^{2}+\tau_{2}(n)\|\boldsymbol{u}\|^{2}\|\boldsymbol{r}\|^{2}\right]\right.$, which takes the value of 1 if and only if the inequality specified in its definition is satisfied and is zero otherwise. Then, $\operatorname{Pd}(n)=\mathrm{E}\left(X_{n}(\boldsymbol{r})\right)$, where the expectation is understood to be with respect to the distribution of $\boldsymbol{r}$ under $H_{1}$. Then, it follows, using the limits specified above, together with the fact that convergence of sets is equivalent to pointwise convergence of indicator set functions [26], $\lim _{n \rightarrow 0} X_{n}(\boldsymbol{r})=\mathrm{I}\left[W(\boldsymbol{r})>\beta\left(\mathrm{Pfa}^{-1 / \alpha}-1\right)\|\boldsymbol{u}\|^{2}\right]$, which is an integrable random variable. Also, $X_{n}(\boldsymbol{r}) \leq 1$ for all $\boldsymbol{r}$, and so $\left|X_{n}(\boldsymbol{r})\right|$ is bounded by an integrable random variable. Hence, Lebesgue's dominated convergence theorem justifies taking the limit inside the expectation in (15), and so

$$
\lim _{n \rightarrow 0} \operatorname{Pd}(n)=\mathrm{P}\left[W(\boldsymbol{r})>\|\boldsymbol{u}\|^{2} \beta\left[\mathrm{Pfa}^{-1 / \alpha}-1\right] \mid H_{1}\right] .
$$

The limit in (16) is the detection probability of the WMF operating in Pareto clutter [18]. This result demonstrates that it is important to not choose $n$ too close to zero; otherwise, the performance of (9) may be similar to the WMF. In the case of horizontally polarised returns, the WMF has been shown to experience a significant detection loss because the clutter is very heavy tailed [16]. Hence, in such cases, it is prudent to select an $n$ further away from zero. For a scenario with vertically polarised returns, this restriction is less important.

As a simple example, suppose we are analysing detection performance with $N=10$ looks and a false alarm probability of $10^{-6}$. Then, $\max \{-6,-3.010\}=-3.010$, which means we need to select an $n>-3.010$. Hence, for the case of detection in vertical polarised clutter, choose for simplicity $n=-1$. Then, $\kappa=10^{5}$ and $\tau_{2}=10^{0.1}-1 \approx$ 0.2589 . If the number of looks is reduced to $N=3$ and the false alarm probability increased to $10^{-4}$, then we need to select an $n$ such that $-0.903<n<0$. For example, in the case of horizontally polarised clutter, we can select $n=-0.5$ so that $\kappa=10^{3.5}$ and $\tau_{2}=10^{0.1667}-1 \approx 0.4678$.

\section{Coherent CFAR detector}

In a practical implementation of the detector (9), it is clear that estimates of the Pareto clutter parameters will need to be used. The most frequently used approach is to apply a maximum likelihood estimation (MLE) process to approximate these unknown parameters. Assuming a moderate to slow scan rate, the MLE should have a sufficient number of data points available to produce clutter estimates. Maximum likelihood estimation for the clutter modelled as a compound Gaussian process with inverse gamma texture has been analysed in [12].

Recently, it has been shown how a property of Pareto order statistics can be used, together with a least squares approach, to estimate the texture clutter parameters [27]. 
This new approach has been shown to improve not only on the accuracy of the MLE procedure, but additionally results in a reduction of computation time. Further, the method works well with sample sizes significantly smaller than those required for accurate estimation with an MLE procedure [27].

An alternative is that it is possible to guess these parameters using the radar's polarisation and azimuth angle as a guide to possible Pareto clutter parameters. Such a crude practice can be justified for the Pareto scale parameter based upon the results in [15], where it is demonstrated that various detectors for the clutter model of interest do not seem to be overly sensitive to this parameter. Hence, bounds can be used to approximate it. For example, it has been found that based upon the Defence Science and Technology Organisation (DSTO) X-band high-resolution radar clutter sets [14], very spiky horizontally polarised returns, in the upwind direction, will generally have very small shape parameters $(3<\alpha<5)$, with scale parameter $\beta<0.1$. For vertically polarised returns, also in the upwind direction, the shape parameter tends to be large $(\alpha>12)$, while the scale parameter $0.1<\beta<1$. Based upon these bounds, we can approximate $\beta$ quite easily. However, a statistical estimation procedure is still required for approximation of the texture shape parameter.

In this work, the focus will not be on the estimation of these parameters but on the cost of approximating them. For example, we will examine the performance of the detector (9) when the Pareto parameters are estimated with a percentage error. This will also be done using Pareto parameters fitted to a number of different $\mathrm{X}$-band radar clutter data sets. Specifically, clutter parameters estimated from the DSTO Ingara radar data [28-30] as well as the Canadian IPIX radar data [12] will be used.

Based upon the detector (9), consider the alternative detector given by

$$
T_{3}(\boldsymbol{r})=\frac{W(\boldsymbol{r})-\hat{\beta}\left(\kappa^{1 / \hat{\alpha}}-1\right)\|\boldsymbol{u}\|^{2}}{\|\boldsymbol{u}\|^{2}\|\boldsymbol{r}\|^{2}}
$$

where $\hat{\alpha}$ and $\hat{\beta}$ are estimates of the respective Pareto clutter parameters. Then, using an analysis as in the derivation resulting in (12), it can be shown that the probability of false alarm for this detector is given by the following:

Lemma 4.1. For the detector (17), operating in a Pareto clutter environment,

$$
\widehat{\mathrm{Pfa}}=\left(\frac{\beta}{\beta+\hat{\beta}\left(\kappa^{1 / \hat{\alpha}}-1\right)}\right)^{\alpha}(1+\tau)^{-N}
$$

The proof is similar to that of the result (10) and so is omitted for brevity.
Due to the fact that estimated clutter parameters are used in the detector (17), the Pfa given by (18) is an estimate. To produce a CFAR detector, a mismatched threshold is used: suppose the threshold is actually set with (13). Further, suppose $\theta \in(0,1)$ is the desired probability of false alarm of the detection scheme. Then, applying the threshold $\tau=(\kappa \theta)^{-1 / N}-1$ to (18), it is immediate that

$$
\widehat{\mathrm{Pfa}_{\mathrm{actual}}}=\left(\frac{\beta}{\beta+\hat{\beta}\left(\kappa^{1 / \hat{\alpha}}-1\right)}\right)^{\alpha} \kappa \theta,
$$

where $\widehat{\mathrm{Pfa}_{\mathrm{actual}}}$ is the resultant estimated false alarm probability due to using a mismatched threshold. The hope is that if the clutter parameter estimates are fairly accurate, there will neither be an increase in the desired false alarm probability nor a significant detection loss.

Observe that when $\kappa=1$, the actual false alarm probability and $\theta$ will match exactly due to the detector (7). An analysis of (19) is now undertaken. To begin, the questions of estimator unbiasedness and standard deviation are addressed. It is immediate that if $\widehat{\alpha}$ and $\widehat{\beta}$ are unbiased estimators of $\alpha$ and $\beta$, respectively, then it is unlikely that (19) is also an unbiased estimator of Pfa (also since $\kappa>1$ ). Table 1 shows Monte Carlo estimates for the mean and standard deviation of (19), for a selection of clutter parameter sets and Pfa. The choice for each $\kappa$ has been on the basis of the result (14) for examples to be considered in the numerical examples to follow in the next section. Each mean and standard deviation estimate has been generated using a Monte Carlo sample size of $10^{5}$, together with the Pareto parameter estimation algorithm developed in [27]. The latter uses a property of the Pareto order statistics, together with a linear regression algorithm. Each estimate of $\alpha$ and $\beta$ is produced using 1,000 sample points, which is explained in [27] to be sufficient. What is immediate from Table 1 is that the estimator (19) has a bias factor; however, the mean is of the correct order. The standard deviation estimates show that the estimator is efficient.

A mathematical analysis is now included to explore properties of (19) further. It is informative to consider the situation where the actual probability of false alarm is smaller than $\theta$. Performing some analysis of (19), this will occur when $\hat{\beta} \kappa^{1 / \hat{\alpha}}-\beta \kappa^{1 / \alpha}>\hat{\beta}-\beta$. Hence, define a function $h(t)=\hat{\beta} t^{1 / \hat{\alpha}}-\beta t^{1 / \alpha}$, for $t \geq 1$. Then, $h(1)=\hat{\beta}-\beta$, and by taking derivatives,

$$
h^{\prime}(t)=t^{-1}\left[\frac{\hat{\beta}}{\hat{\alpha}} t^{1 / \hat{\alpha}}-\frac{\beta}{\alpha} t^{1 / \alpha}\right] .
$$

Observe that the derivative in (20) is positive when

$$
t>\left(\frac{\widehat{\alpha} \beta}{\alpha \widehat{\beta}}\right)^{\frac{\alpha \widehat{\alpha}}{\alpha-\widehat{\alpha}}},
$$


Table 1 Mean and standard deviation of $\widehat{P f a}$

\begin{tabular}{cccccc}
\hline $\boldsymbol{\alpha}$ & $\boldsymbol{\beta}$ & $\boldsymbol{\kappa}$ & $\boldsymbol{\theta}$ & Mean & Standard deviation \\
\hline 15.8983 & 0.1812 & $10^{4.99}$ & $10^{-5}$ & $1.2297 \times 10^{-5}$ & $6.6180 \times 10^{-6}$ \\
15.8983 & 0.1812 & $10^{6.5}$ & $10^{-7}$ & $1.3865 \times 10^{-7}$ & $1.0199 \times 10^{-7}$ \\
3.4653 & 0.0029 & $10^{3}$ & $10^{-4}$ & $1.0907 \times 10^{-4}$ & $3.2809 \times 10^{-5}$ \\
3.4653 & 0.0029 & $10^{5}$ & $10^{-6}$ & $1.2232 \times 10^{-6}$ & $6.4658 \times 10^{-7}$ \\
1.5582 & $4.3286 \times 10^{-4}$ & $10^{2}$ & $10^{-5}$ & $1.0458 \times 10^{-5}$ & $1.9906 \times 10^{-6}$ \\
1.5582 & $4.3286 \times 10^{-4}$ & $10^{2}$ & $10^{-6}$ & $1.0444 \times 10^{-6}$ & $1.9879 \times 10^{-7}$ \\
1.8587 & 0.001 & $10^{4.5}$ & $10^{-5}$ & $1.1831 \times 10^{-5}$ & $5.5198 \times 10^{-6}$ \\
1.8587 & 0.001 & $10^{3}$ & $10^{-4}$ & $1.0899 \times 10^{-4}$ & $3.2448 \times 10^{-5}$ \\
\hline
\end{tabular}

provided $\alpha>\widehat{\alpha}$. Thus, it is required that $\frac{\widehat{\alpha} \beta}{\alpha \hat{\beta}} \leq 1$. Hence, assuming these conditions hold, it follows that $h$ is an increasing function for $t \geq 1$, and so $h(t) \geq h(1)$ for all $t \geq 1$, and the estimated actual probability of false alarm will be smaller than $\theta$.

An alternative condition can be established by introducing the function

$$
f(t)=\frac{t}{\left[1+\omega\left(t^{1 / \widehat{\alpha}}-1\right)\right]^{\alpha}},
$$

where $\omega=\widehat{\beta} / \beta$. Note that $f(1)=1$, and so in view of (19), the estimated actual probability of false alarm will be smaller than $\theta$ when $f$ is a decreasing function. By applying the quotient differentiation rule, it can be shown that

$$
f^{\prime}(t)=\frac{1-\omega+(\omega-\delta) t^{1 / \widehat{\alpha}}}{\left[1+\omega\left(t^{1 / \widehat{\alpha}}-1\right)\right]^{\alpha+1}},
$$

where $\delta=\frac{\alpha \widehat{\beta}}{\alpha \beta}$. Note that the denominator in (22) is always positive because $t \geq 1$. In the case where $\delta \geq 1$, the derivative is bounded by

$$
f^{\prime}(t) \leq \frac{(\omega-1)\left(t^{1 / \widehat{\alpha}}-1\right)}{\left[1+\omega\left(t^{1 / \widehat{\alpha}}-1\right)\right]^{\alpha+1}}<0,
$$

when $\omega<1$, since $t>1$.

To summarise these results, the estimated resultant probability of false alarm (19) is smaller than the desired probability of false alarm $\theta$ provided the condition

$$
\frac{\alpha}{\hat{\alpha}} \frac{\hat{\beta}}{\beta} \geq 1
$$

holds, together with one of the requirements

(i) $\hat{\alpha}<\alpha$

(ii) $\hat{\beta}<\beta$.

In a practical implementation of the detector (17), using the threshold based upon (13), the hope is that if these condition are valid, we can achieve approximate CFAR control, with at worst a reduction in the desired false alarm rate. Observe that condition (26) justifies the usage of a lower bound on the Pareto scale parameter.

Note that in the case where condition (25) holds, then if $\hat{\beta}>\beta$, it is immediate that (24) is valid. Hence, underestimating the shape parameter is necessary in practice in order to ensure that the false alarm probability does not increase. This means it will be required to utilise lower bounds on data, coupled with an MLE procedure. The practical implementation of such a scheme, relative to the DSTO Ingara data, will be explored in subsequent research.

As some examples when these conditions are met in practice, suppose we underestimate the Pareto shape parameter with a $10 \%$ error, and the Pareto scale parameter is overestimated by $10 \%$. By this, it is understood that $\widehat{\alpha}=0.9 \alpha$ and $\widehat{\beta}=1.1 \beta$. Then, the conditions (24) and (25) are both met. If both parameters are underestimated by the same percentage error, then (24) will hold, as well as both (25) and (26). If the shape parameter is underestimated with an error of $10 \%$, while the scale parameter is underestimated with a $5 \%$ error, then all three conditions will also hold.

\section{Performance analysis of detectors}

Performance assessment of the two detectors (9) and (17) is now undertaken by examining curves plotting the probability of detection (Pd) as a function of the signal-toclutter ratio (SCR), for a fixed probability of false alarm. As a comparison, the performance of the LTD (5) and 
WMF (6) are included. Additionally, the NMF (7) is also analysed since it is a special case of the detector (9), which has no requirement for Pareto clutter parameter estimation. In all cases, the detection probability has been estimated using Monte Carlo simulation, with $10^{6}$ runs. The clutter is assumed to have an exponential correlation structure, also known as a Toeplitz form, as in [8] and also [18]. This means we assume $\Sigma(i, j)=\rho^{|i-j|}$, where $0<\rho<1$ and the indices $i$ and $j$ range from 1 to the maximum number of looks. In addition, the normalised Doppler frequency has been fixed at 0.5 throughout. A simple Gaussian target model has also been employed in all examples, as in $[2,18]$, which is also known colloquially in the signal processing literature as a Swerling I target model [2].

As remarked previously, clutter parameters will be based upon estimates obtained using MLE on real data sets. The Australian DSTO Ingara data set is a series of pure clutter returns obtained using the Ingara radar during a trial in the Southern Ocean in 2004. The Ingara radar is an airborne X-band, fully polarimetric radar, which operated in a circular spotlight mode. Further details concerning this radar can be found in [30], while a thorough analysis of the data is reported in [28]. During the data gathering exercise, the radar surveyed the same ocean patch at different azimuth angles, operating at $10.1 \mathrm{GHz}$ with a $20-\mu$ s compressed pulse width, with a range resolution of $0.75 \mathrm{~m}$. The fitting of a Pareto intensity model to the Ingara data is described in [14].

The IPIX radar is an experimental X-band search radar belonging to Canada's McMaster University [31,32], and a clutter collection exercise was undertaken in 1998 as reported in [12]. As detailed in the latter, this radar is capable of dual polarised operations and used a dual frequency transmission range of between 8.9 and $9.4 \mathrm{GHz}$, with a pulse width of $0.06 \mu \mathrm{s}$. The range resolution is reported to have been $9 \mathrm{~m}$. The clutter trial was conducted at a fixed location observing Lake Ontario from a height of $20 \mathrm{~m}$ so that the radar was stationary. Clutter parameter estimates are reported in [12], which provide a completely different range of Pareto clutter parameters than those obtained from the Ingara radar.

In the analysis to follow, four data sets will be used to present the performance of the detectors under a number of different scenarios. With reference to radar polarisation, throughout, $\mathrm{HH}$ will represent horizontal transmit and receive, and VV will be the vertical transmit and receive case. No examples of cross polarisation are included due to the fact that clutter parameter estimates resulting from this polarisation tend to fall between those obtained from the $\mathrm{HH}$ and VV polarisations [18]. Each example is listed by radar, polarisation and clutter parameter estimates. The definition of the inverse gamma distribution in [12] replaces $\beta$ with its reciprocal in (3), which has been taken into account in the IPIX-based examples to follow.

\subsection{Ingara, $\mathrm{VV}, \alpha=\mathbf{1 5 . 8 9 8 3}, \beta=\mathbf{0 . 1 8 1 2}$}

The first example is based upon a typical VV-polarised clutter set obtained from the Ingara data trial. For this particular data set, the Pareto clutter parameters have been estimated to be $\alpha=15.8983$ and $\beta=0.1812$. Additionally, this clutter set was obtained at an azimuth angle of $255^{\circ}$ (upwind direction is approximately $227^{\circ}$ ). Figure 1 shows two plots of the performance of the WMF, LTD and NMF when the clutter parameters are assumed to be known. The performance of the detector (9) is also included and is referred to as the alternative NMF (ANMF) in the plots. The left subplot is for the case where the number of looks is $N=5$, the Toeplitz factor is $\rho=0.8$ and a probability of false alarm is $10^{-5}$. With reference to (14), since $\max \{-5,-1.505\}=-1.505$ and we are examining the VV-polarised case, we select $n=-0.01$ so that $\kappa=10^{4.99}$. The plot shows the WMF and ANMF matching very closely, while the LTD has a detection loss relative to these detectors. This is due to the fact that the clutter is approximately Gaussian distributed. The NMF saturates because of the small number of looks. Hence, in this case, we see that the design of the ANMF has improved detection performance in the case of a small number of looks.

The right subplot in Figure 1 is for the same clutter, but when $N=8, \rho=0.8$ and the false alarm probability is set to $10^{-7}$. Hence, since $\max \{-7,-2.408\}=-2.408$, we select $n=-0.5$ to generate $\kappa=10^{6.5}$. Here, we observe a similar situation to the previous plot, except that the LTD has a larger detection loss relative to the WMF and ANMF. The NMF has again saturated.

Figure 2 examines the performance of the detector (17), relative to the four detectors WMF, LTD, NMF and ANMF, where the latter detectors have full knowledge of clutter parameters. In the figures to follow, detector (17) will be abbreviated to ANMF Approx. In this simulation, the left subplot is for $N=20$ and $\rho=0.5$, and the false alarm probability has been set to $10^{-6}$. Since $\max \{-6,-6.02\}=-6, n=-5$ has been selected so that $\kappa=10$. It is assumed that $\widehat{\alpha}$ has been overestimated by double, while $\widehat{\beta}$ has been underestimated by $20 \%$. By using (19), it can be shown the resultant $\mathrm{Pfa}=3.955 \times 10^{-6}$, yielding an increase in the false alarm rate. All the other detectors have had their thresholds set so that they have this same resultant false alarm probability to provide a valid comparison. The left subplot in Figure 2 shows that the WMF has the best performance, followed by the LTD and then the ANMF. The detector (17) has better performance than the NMF, despite the fact that it requires parameter estimation. 


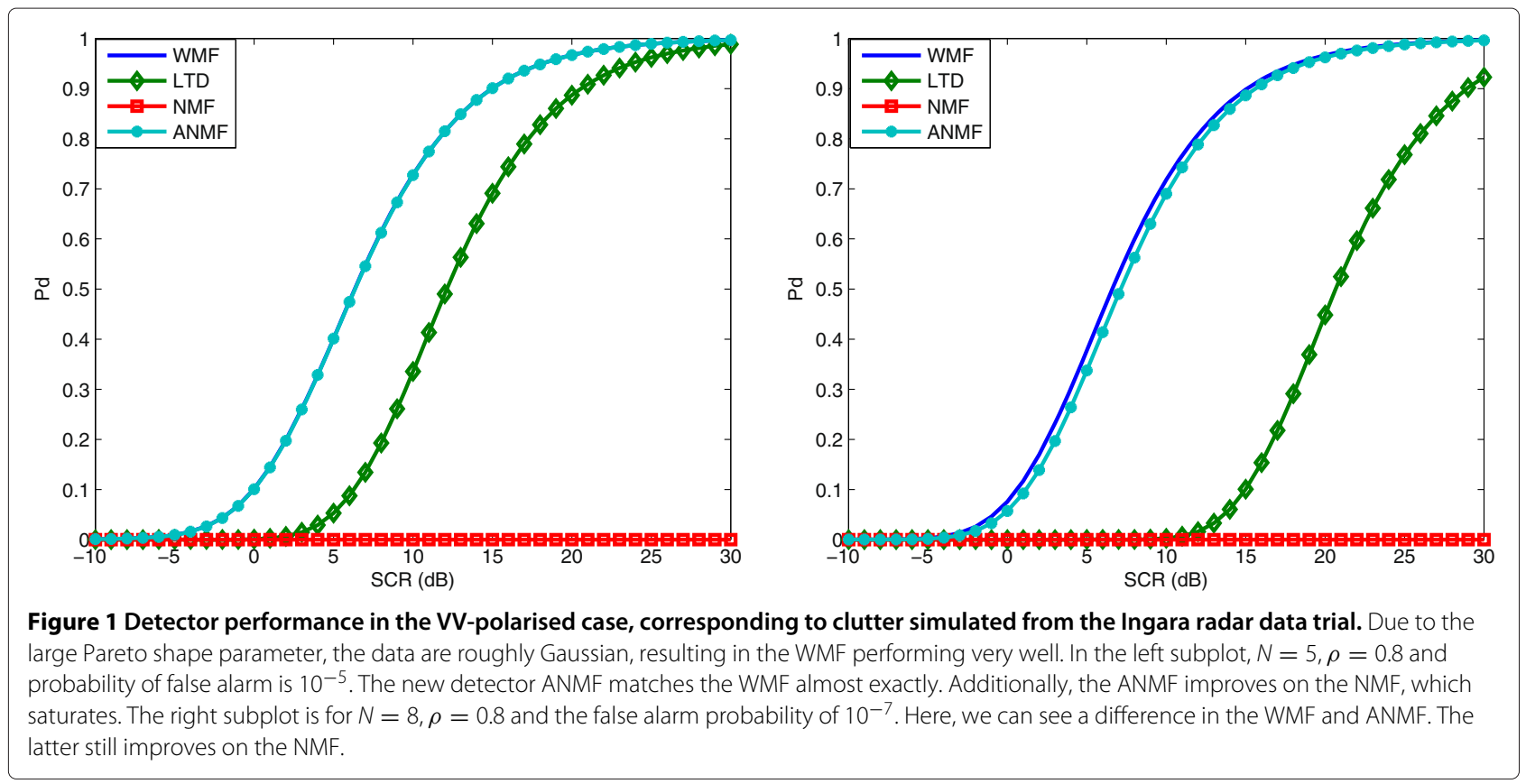

The right subplot in Figure 2 is for the case where $N=10, \rho=0.5$ and false alarm probability is $10^{-7}$. Noting that $\max \{-7,-3.01\}=-3.01, n=-1$ has been selected, and consequently, $\kappa=10^{6}$. In this example, it is assumed that the shape parameter estimate is overestimated by triple, while the scale parameter is also overestimated, but by double. The subplot shows the ANMF matching the WMF very closely, while the estimator (17) has better performance than the LTD. The NMF has completely saturated due to an insufficient number of looks. In this example, the resultant false alarm probability is $2.8255 \times 10^{-5}$, which has been used to set the thresholds for the four other detectors. What is observed here is an increase in the false alarm probability due to severe inaccuracy in the clutter parameter estimates.

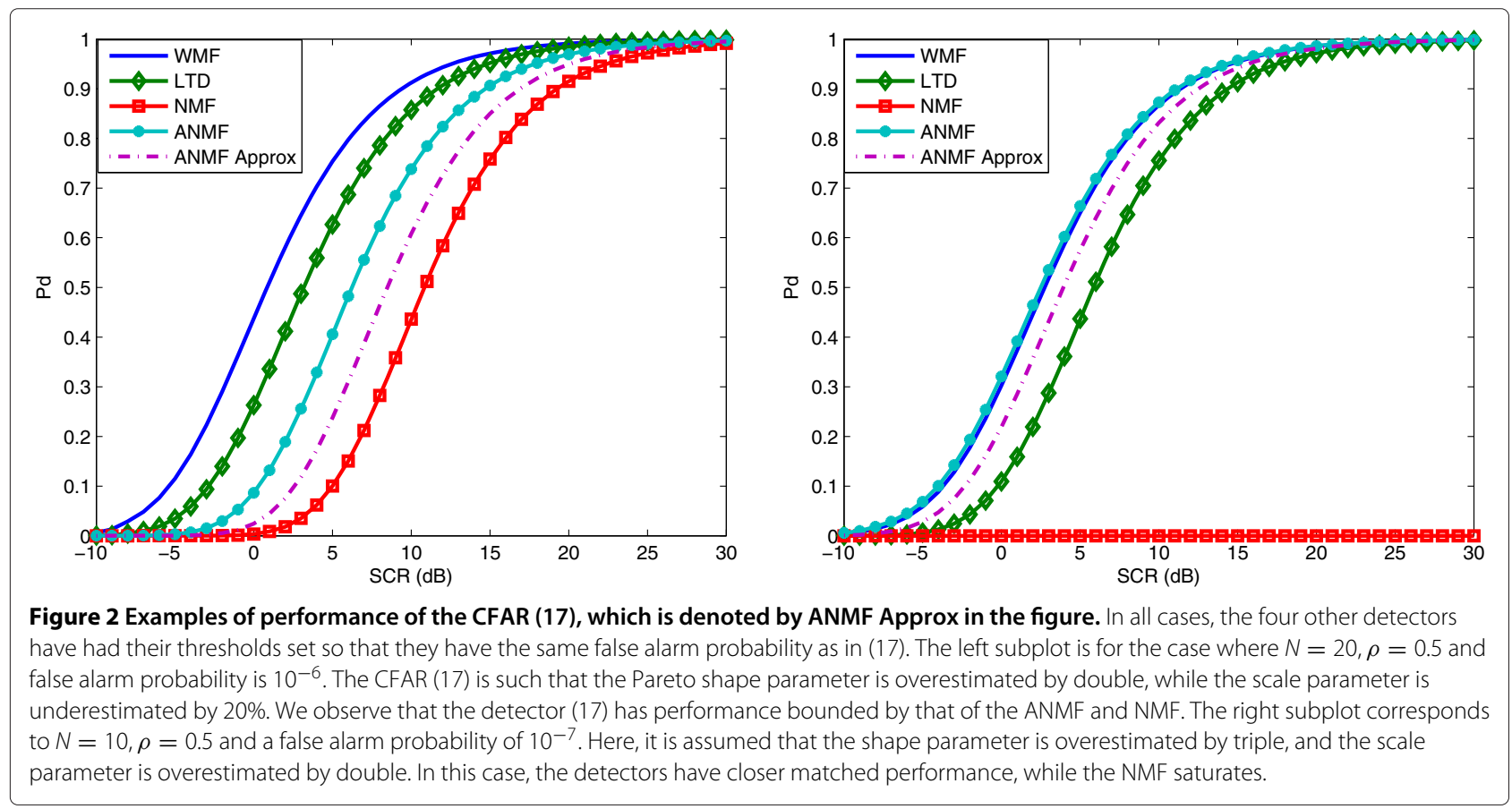



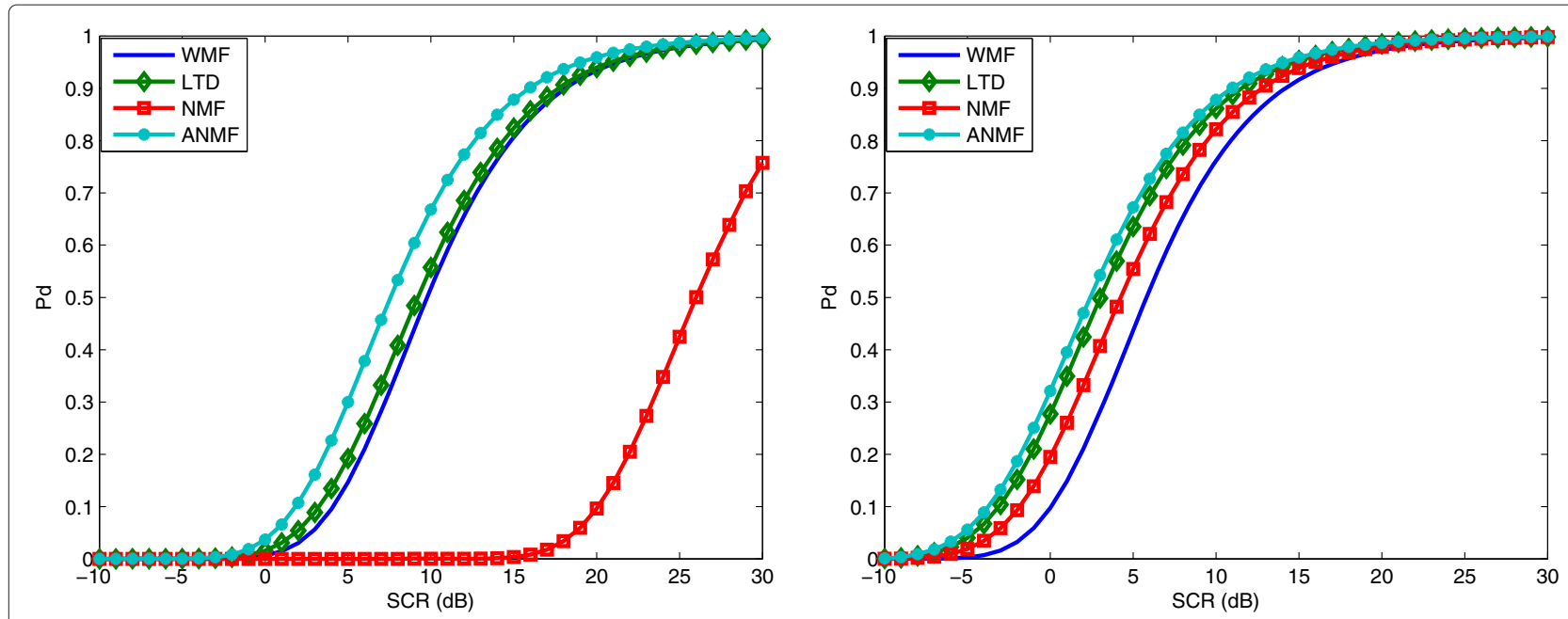

Figure 3 Performance analysis in HH-polarised clutter, based upon the Ingara data. The left subplot is for $N=20, \rho=0.1$ and a false alarm probability of $10^{-6}$. This example shows that the ANMF can work very well, and certainly improves on the NMF. The right subplot is for $N=25, \rho=0.1$ and false alarm probability of $10^{-5}$. In this scenario, we observe the ANMF provides a small gain on the LTD.

\subsection{Ingara, $\mathrm{HH}, \alpha=3.4653, \beta=0.0029$}

The second example is for the case of $\mathrm{HH}$-polarised clutter, again obtained from the Ingara data trial. In this case, the azimuth angle was $315^{\circ}$, and the Pareto clutter parameters are $\alpha=3.4653$ and $\beta=0.0029$. Figure 3 shows detector performance when the texture parameters are known. The left subplot is for $N=20, \rho=0.1$ and a false alarm probability of $10^{-6}$. Since $\max \{-6,-6.02\}=$ -6 , we have selected $n=-1$ so that $\kappa=10^{5}$. With this choice, it is observed that the ANMF introduces a detection gain over the LTD and the WMF. Additionally, the performance of the ANMF far exceeds that of the NMF, which has poorer performance than the WMF.

The right subplot in Figure 3 is for $N=25, \rho=0.1$ and false alarm probability of $10^{-5}$. Since $\max \{-5,-7.525\}=$ $-5, n=-4$ has been selected, and hence, $\kappa=$ 10. Increasing the number of looks has resulted in an improvement on the performance of the NMF, which introduces a detection gain on the WMF. The ANMF has the best performance, with a slight gain on the LTD.

Figure 4 shows some examples of the performance of the detector (17) in horizontally polarised clutter. The left subplot corresponds to $N=30, \rho=0.5$ and a probability of false alarm of $10^{-6}$. Due to $\max \{-6,-9.03\}=-6$, $n=-5$ has been selected so that $\kappa=10$. It is assumed that both texture parameters have been overestimated by a factor of 5; consequently, the resultant false alarm probability is $3.0169 \times 10^{-8}$, based upon (19). Hence, there is a large reduction in the false alarm probability. All other detectors have had their thresholds set relative to this resultant probability. The subplot shows that the WMF has very poor performance, as expected in heavy-tailed clutter. The ANMF and LTD have very good performance, with the ANMF having slightly larger probability of detection. The ANMF approximation (17) has very good performance, despite the inaccuracy with its parameter estimates. In fact, it is slightly better than the LTD. The NMF's performance trails that of the LTD. This example shows that the new CFAR (17) can improve on the NMF considerably.

The right subplot in Figure 4 is for $N=40, \rho=$ 0.9 and a false alarm probability of $10^{-7}$. Due to the fact that $\max \{-7,-12.04\}=-7, n=-6$ has been selected so that $\kappa=10$ as previously. Here, it is assumed that the clutter parameter $\alpha$ is overestimated by double, while there is a $20 \%$ error in the estimation of $\beta$. The resultant false alarm probability is $3.0169 \times 10^{-8}$, which has resulted in an order of magnitude reduction in the desired false alarm rate. The subplot shows similar results to the previous subplot, except that all detectors have closer performance, with the exception of the WMF. The CFAR (17) is matching the performance of the LTD almost exactly in this case.

\subsection{IPIX, HH, $\alpha=\mathbf{1 . 5 5 8 2}, \beta=4.3286 \times 10^{-4}$}

Clutter parameters are now sourced from the IPIX radar trial. For this example, we take the case of $\mathrm{HH}$-polarised returns, using the Pareto clutter parameters $\alpha=1.5582$ and $\beta=4.3286 \times 10^{-4}$. It is interesting to note that the IPIX clutter parameters are much smaller than those obtained from the Ingara data trial. Such a small shape parameter indicates very spiky returns. Figure 5 shows detector performance when both texture parameters are known. The left subplot corresponds to the case where 


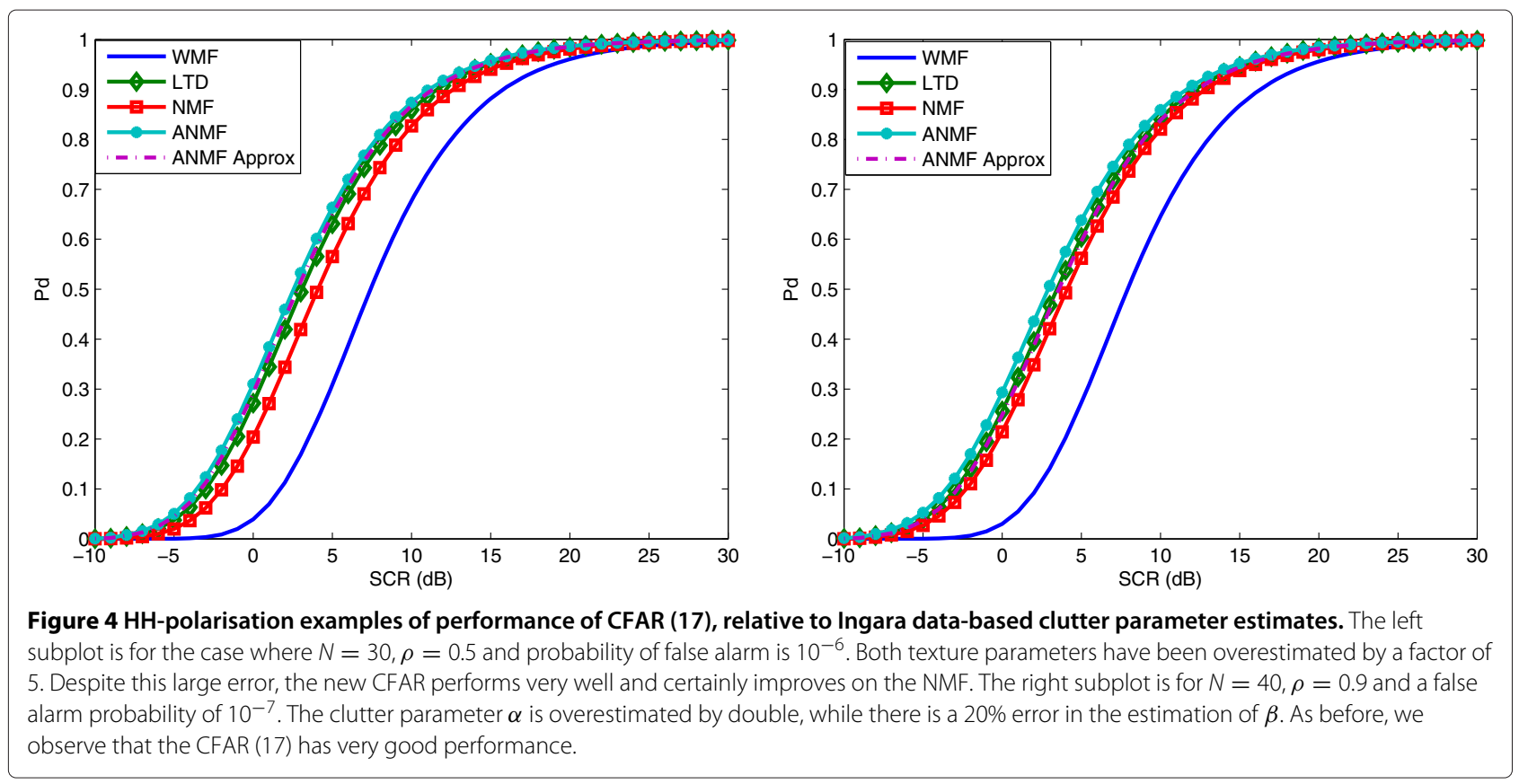

$N=30, \rho=0.4$ and false alarm probability is $10^{-6}$. Since $\max \{-6,-9.03\}=-6, n=-4$ has been selected and $\kappa=100$. The subplot shows that the WMF has very poor performance. The ANMF improves on the performance of both the LTD and NMF.

The right subplot corresponds to $N=25, \rho=$ 0.7 and probability of false alarm of $10^{-5}$. Due to $\max \{-5,-7.525\}=-5, n=-4$ has been chosen and so $\kappa=10$. The results are similar to the previous subplot, except that the performance of the ANMF is much more pronounced. The ANMF is better than the LTD and NMF as before.

Figure 6 shows the performance of the CFAR (17) relative to the other detectors. The left subplot is for $N=$ $30, \rho=0.4$ and a false alarm probability of $10^{-6}$. Since $\max \{-6,-9.03\}=-6, n=-4$ has been selected so $\kappa=100$. For this simulation, it is assumed that the shape parameter $\alpha$ has been overestimated by $50 \%$, while the scale parameter is overestimated by 5 times its actual value. This results in an actual false alarm probability of $4.5453 \times 10^{-7}$, which is a reduction in the desired false alarm rate. The subplot shows that the WMF has very poor performance, which is due to the very spiky nature of the clutter. The CFAR (17) has performance bounded above by that of the ANMF and bounded from below by the LTD. Detector NMF has smaller probability of detection than LTD. In this example, the CFAR (17) has very good performance. All detectors have the same probability of false alarm.

The right subplot is for $N=25, \rho=0.7$ and a false alarm probability of $10^{-7}$. Since $\max \{-7,-7.525\}=$ $-7, n=-5$ has been selected, and hence, $\kappa=100$.
Both clutter parameters are assumed to be underestimated by $20 \%$. The resultant false alarm probability can be shown to be $4.4342 \times 10^{-8}$, which is a reduction in the design false alarm rate. The subplot shows that the ANMF has the best performance, and the CFAR (17) introduces only a tiny detection loss relative to the ANMF. The LTD has a significant detection loss relative to the ANMF and its approximation. The NMF also has a significant detection loss relative to the ANMF, (17) and the LTD. The WMF has very poor performance in this example.

\subsection{IPIX, VV, $\alpha=1.8587, \beta=0.001$}

The final example is for a VV-polarised case based upon the IPIX radar trial. In this scenario, the clutter parameters are $\alpha=1.8587$ and $\beta=0.001$. Although this example is for the VV channel, these clutter parameters indicate very heavy-tailed non-Gaussian clutter statistics [33]. Figure 7 shows detector performance under the assumption of known clutter parameters. The left subplot is for $N=30, \rho=0.6$ and a false alarm probability of $10^{-5}$. Since $\max \{-5,-9.03\}=$ $-5, n=-3$ has been selected, so $\kappa=100$. The subplot shows that the WMF has very poor performance. The ANMF has better performance than the LTD and NMF.

The right subplot in Figure 7 is for $N=25, \rho=$ 0.1 and a false alarm probability of $10^{-7}$. Due to $\max \{-7,-7.525\}=-7, n=-6$ has been chosen, with $\kappa=10$. The subplot shows that the ANMF has very good performance relative to the LTD and NMF, while the 


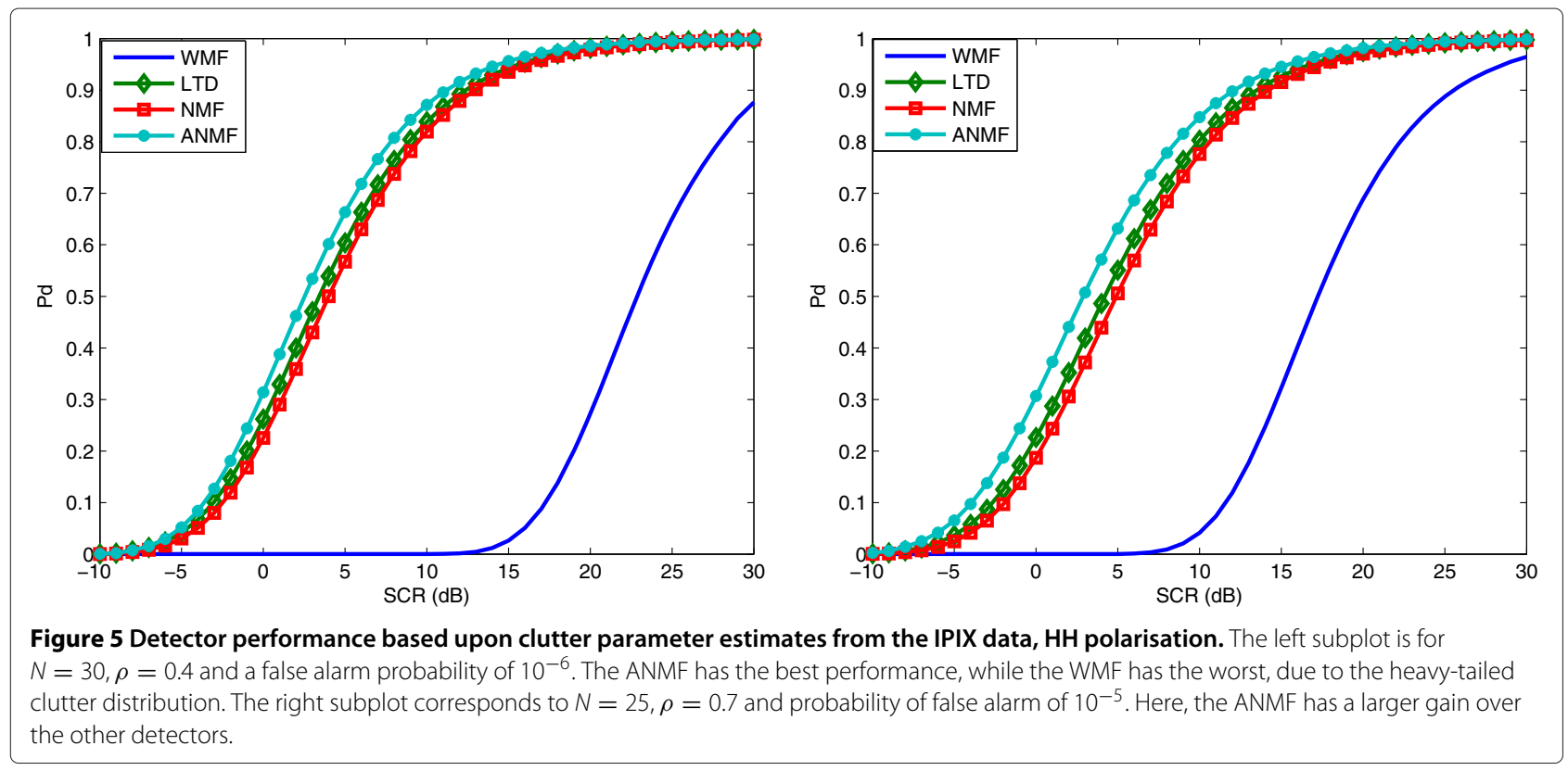

WMF has very poor performance. This is another example showing that the ANMF works very well in spiky clutter.

Figure 8 now examines the performance of the CFAR (17) in this clutter scenario. The left subplot is for $N=$ $40, \rho=0.6$ and a false alarm probability of $10^{-5}$. In view of the fact that $\max \{-5,-12.04\}=-5, n=-4$ has been selected so that $\kappa=10$. It is assumed that the parameter $\alpha$ is underestimated by $20 \%$, while $\beta$ is overestimated by $20 \%$. This results in an actual false alarm probability of $4.2849 \times 10^{-6}$, which is a decrease in the desired false alarm rate level. The subplot shows that the ANMF and its CFAR approximation (17) have very comparable performance, with both detectors performing better than the LTD and NMF. Here, as before, the WMF has the worst performance. All detectors are operating with the same false alarm probability, for consistency.

The right subplot is for $N=30, \rho=0.1$ and a false alarm probability of $10^{-7}$. Since $\max \{-7,-9.03\}=-7$, $n=-6$ has been selected, yielding $\kappa=10$. For this example, the Pareto shape parameter is overestimated by $80 \%$,
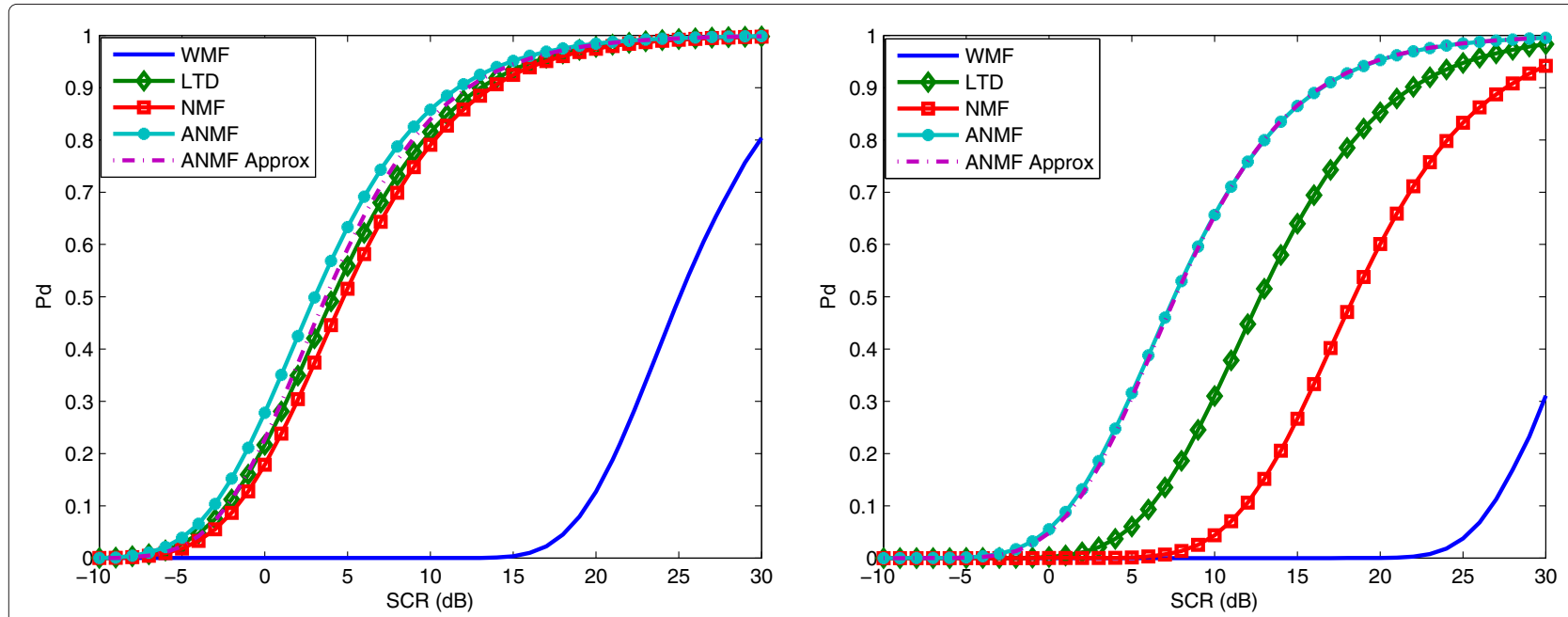

Figure 6 Performance of the CFAR detector (17) in HH-polarised IPIX trial-based clutter. The left subplot is for $N=30, \rho=0.4$ and a false alarm probability of $10^{-6}$. It has been assumed that the shape parameter $\alpha$ has been overestimated by $50 \%$, while the scale parameter is overestimated by 5 times its actual value. The plot shows that the new CFAR has very good performance despite parameter estimation inaccuracy, The right subplot is for $N=25, \rho=0.7$ and a false alarm probability of $10^{-7}$. Both clutter parameters are assumed to be underestimated by $20 \%$. Here, we observe that the CFAR (17) has a very small loss relative to the ANMF, and both these two new detectors have the best performance. 

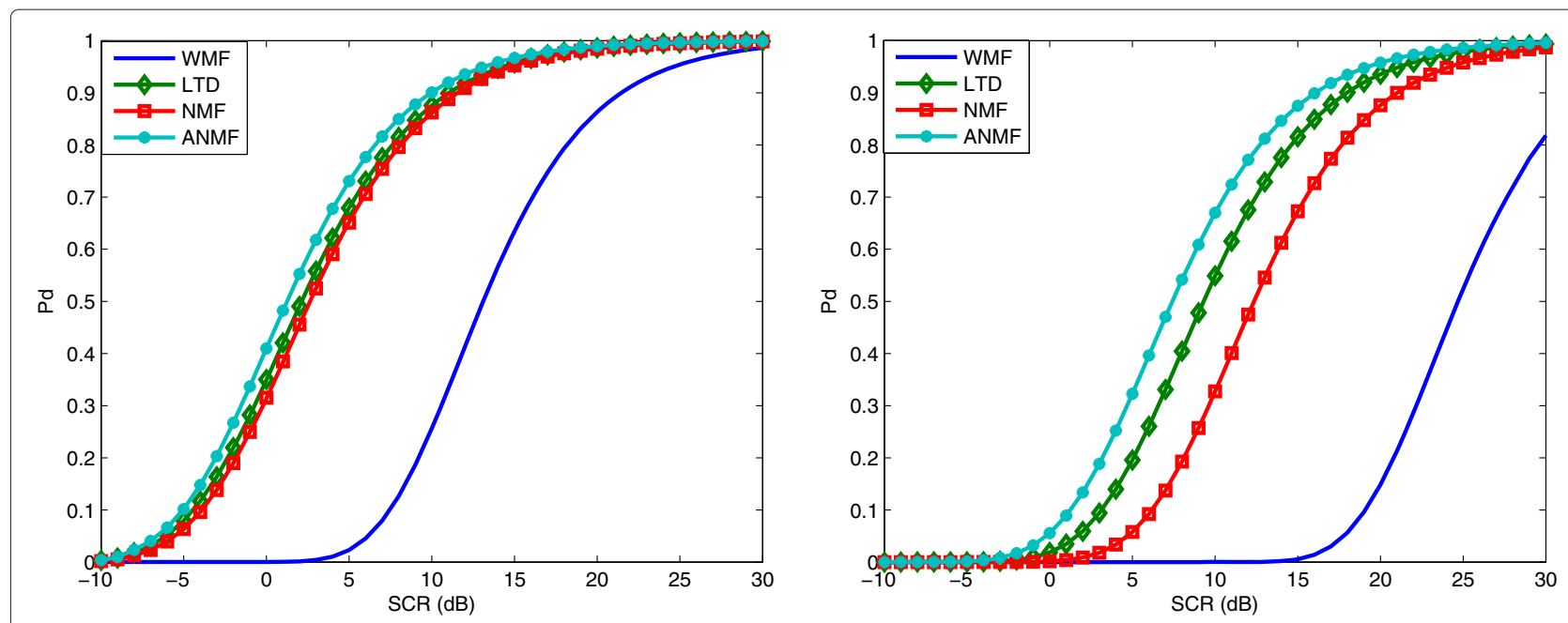

Figure 7 Detector performance with clutter parameter estimates based upon the IPIX radar trial, with VV polarisation. The left subplot is for $N=30, \rho=0.6$ and a false alarm probability of $10^{-5}$. The ANMF has a gain over all other detectors. The right subplot is for $N=25, \rho=0.1$ and a false alarm probability of $10^{-7}$. Again, the ANMF has very good performance. Due to the heavy-tailed nature of the clutter, the WMF has very poor performance.

while the scale parameter is overestimated by $20 \%$. This results in an actual false alarm probability of $2.3328 \times$ $10^{-7}$, which is larger than the desired false alarm rate. The plot shows similar results to the previous subplot, except that it is easier to discern the differences in detector performance. Again, the ANMF has the best performance, and the CFAR (17) has very good performance.

\section{Conclusions}

This paper introduced a modification of the NMF detector, referred to as the ANMF and given by (9), which was shown to work very well in practice. By using a parameter $\kappa$, which is a function of the design false alarm probability and the number of looks, and whose value is then determined by an application of (14), one can avoid the detector saturation issues inherent in the NMF. This new detector was shown to outperform the LTD and WMF in a large number of cases, when clutter parameters are known. In particular, this tended to occur for the case where the number of looks was large $(N>>30)$, and the clutter is very spiky. In addition to this, by then introducing approximations to the clutter parameters, an approximate
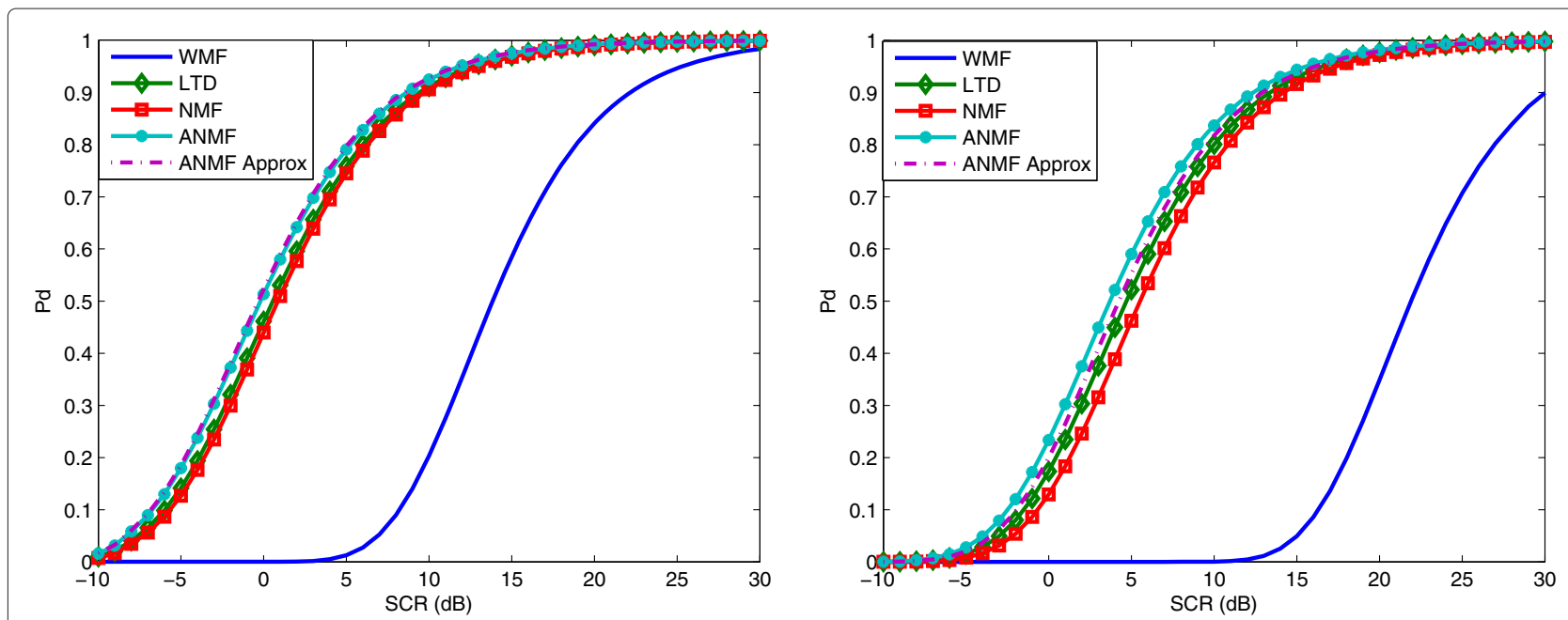

Figure 8 Analysis of the CFAR detector (17) in the same clutter environment as for Figure 7. The left subplot is for the case where $N=40, \rho=0.6$ and false alarm probability is $10^{-5}$. It is assumed that the parameter $\alpha$ is underestimated by $20 \%$, while $\beta$ is overestimated by $20 \%$. We see that the new CFAR has very good performance. The right subplot is for $N=30, \rho=0.1$ and a false alarm probability of $10^{-7}$. For this example, the Pareto shape parameter is overestimated by $80 \%$, while the scale parameter is overestimated by $20 \%$. As before, the new CFAR has excellent performance. 
CFAR version of this detector was analysed. It was shown to perform very well in practice, depending on the error in the approximation of the clutter parameters. The error in clutter parameter estimation resulted in a change in the desired false alarm probability. Some conditions were established to ascertain when there was not an increase in the false alarm rate. Provided that the clutter parameter errors were small, there were only small variations in the false alarm rate, as expected. Despite this, the CFAR (17) was shown to have very good performance in practice.

In further research, the detector (17) will be applied to real data to assess its performance in practice. This will be coupled together with real-time parameter estimation based upon the work of [27].

\section{Abbreviations}

ANMF: Alternative normalised matched filter; CFAR: Constant false alarm rate; DSTO: Defence Science and Technology Organisation; GLRT: Generalised likelihood ratio test; $\mathrm{HH}$ : Horizontal transmit and receive; LTD: Linear threshold detector; MLE: Maximum likelihood estimation; NMF: Normalised matched filter; Pd: Probability of detection; Pfa: Probability of false alarm; SCR: Signal-to-clutter ratio; SIRP: Spherically invariant random process; W: Vertical transmit and receive; WMF: Whitening matched filter.

\section{Authors' contributions}

The author declares that there are no competing interests.

\section{Acknowledgements}

The author would like to express thanks to an anonymous reviewer whose comments significantly improved the content and results of this paper.

Received: 8 January 2013 Accepted: 2 May 2013

Published: 15 May 2013

\section{References}

1. KJ Sangston, F Gini, in IEEE Radar Conference. MS Greco, New results on coherent radar target detection in heavy-tailed compound Gaussian clutter, Washington, DC, 2010), pp. 779-784. 10-14 May

2. KJ Sangston, F Gini, MS Greco, Coherent radar target detection in heavy-tailed compound Gaussian clutter. IEEE Trans. Aerosp. Electron. Syst. 48, 64-77 (2012)

3. E Conte, M Longo, Characterisation of radar clutter as a spherically invariant random process. IEE Proc, Pt. F: Commun. Radar Signal Process 134, 191-197 (1987)

4. M Rangaswamy, D Weiner, A Ozturk, Non-Gaussian random vector identification using spherically invariant random processes. IEEE Trans. Aerosp. Elec. Syst. 29, 111-123 (1993)

5. KJ Sangston, K Gerlach, Coherent detection of radar targets in a non-Gaussian background. IEEE Trans. Aerosp. Electron. Syst. 3, 330-340 (1994)

6. F Gini, Suboptimal coherent radar detection in a mixture of K-distributed and Gaussian clutter. IEE Proceed. Part F: Radar, Sonar, Navig. 144, 39-48 (1997)

7. F Gini, MV Greco, A Farina, P Lombardo, Optimum and mismatched detection against K-distributed plus Gaussian clutter. IEEE Trans. Aerosp. Electron. Syst. 34, 861-876 (1998)

8. F Gini, MV Greco, Suboptimal approach to adaptive coherent radar detection in compound Gaussian clutter. IEEE Trans. Aerosp. Electron. Syst. 35, 1095-1104 (1999)

9. E Conte, M Lops, G Ricci, Asymptotically optimum radar detection in compound Gaussian clutter. IEEE Trans. Aerosp. Elec. Sys. 31, 617-625 (1995)

10. Y Dong, Optimal coherent radar detection in a K-distributed clutter environment. IET Radar, Sonar Navig. 6, 238-292 (2012)

11. GV Weinberg, in Digital Communication, ed. by C Palanisamy. Coherent multilook radar detection for targets in KK-distributed clutter (Intech, Croatia, 2012)
12. A Balleri, A Nehorai, J Wang, Maximum likelihood estimation for compound-Gaussian clutter with inverse-gamma texture. IEEE Trans. Aerosp. Electron. Syst. 43, 775-780 (2007)

13. M Farshchian, FL Posner, in IEEE Radar Conference. The Pareto distribution for low grazing angle and high resolution $\mathrm{X}$-band sea clutter, Washington, DC, 2010), pp. 789-793. 10-14 May

14. GV Weinberg, Assessing the Pareto fit to high resolution high grazing angle sea clutter. IET Electron. Lett. 47, 516-517 (2011)

15. X Shang, H Song, Radar detection based on compound-Gaussian model with inverse gamma texture. IET Radar, Sonar, Navig. 5, 315-321 (2011)

16. GV Weinberg, Coherent multilook radar detection for targets in Pareto distributed clutter. IET Electron. Lett. 47, 822-824 (2011)

17. P Stinco, M Greco, F Gini, Adaptive detection in compound-Gaussian clutter with inverse gamma texture. IEEE Int. Conf. Radar. 1, 434-437 (2011)

18. GV Weinberg, Assessing detector performance, with application to Pareto coherent multilook radar detection. IET Radar, Sonar, Navig. 7 (2013) (in press)

19. GP Beaumont, Intermediate Mathematical Statistics. (Chapman and Hall, London, 1980)

20. G Minkler, J Minkler, CFAR: The Principles of Automatic Radar Detection in Clutter. (Magellan, Baltimore, 1990)

21. J Guan, YN Peng, Y He, Proof of CFAR by the use of the invariant test. IEEE Trans. Aerosp. Elec. Sys. 36, 336-339 (2000)

22. KJ Sangston, F Gini, MV Greco, A Farina, Structures for radar detection in compound Gaussian clutter. IEEE Trans. Aerosp. Elec. Sys. 35, 445-458 (1999)

23. R Ravid, N Levanon, Maximum-likelihood CFAR for Weibull background IEE Proceed. F. 139, 256-264 (1992)

24. V Anasstassopoulos, GA Lampropoulos, Optimal CFAR detection in Weibull clutter. IEEE Trans. Aerosp. Elec. Sys. 31, 52-64 (1995)

25. P Billingsley, Probability and Measure (2nd Ed). (Wiley, New York, 1986)

26. RG Kuller, Topics in Modern Analysis. (Prentice-Hall, Englewood Cliffs, 1969)

27. GV Weinberg, Estimation of Pareto clutter parameters using order statistics and linear regression. IET Electron. Lett (2013). (in press)

28. Y Dong, Distribution of $X$-band high resolution and high grazing angle sea clutter (DSTO Technical Report RR-0316,2006) (2013). http://www. dtic.mil/cgi-bin/GetTRDoc?AD=ADA462947, Accessed 12 May

29. N Stacy, D Crisp, A Goh, D Badger, M Preiss, Polarimetric analysis of fine resolution X-band sea clutter data. Proceed. Int. Geoscience Remote Sensing Symp. 4, 2787-2790 (2005)

30. NJS Stacy, MP Burgess, Ingara: the Australian airborne imaging radar system. Proceed. Int. Geoscience Remote Sensing Symp. 4, 2240-2242 (1994)

31. TJ Nohara, S Haykin, Canadian east coast radar trials and the K-distribution. IEE Proc. F. 138, 80-88 (1991)

32. A Farina, F Gini, MV Greco, L Verrazzani, High resolution sea clutter data: statistical analysis of recorded live data. IEE Proc. -Radar, Sonar, Navig. 144, 121-130 (1997)

33. GV Weinberg, Validity of whitening matched filter approximation to the Pareto coherent detector. IET Sig. Proc. 6, 546-550 (2012)

doi:10.1186/1687-6180-2013-105

Cite this article as: Weinberg: Coherent CFAR detection in compound Gaussian clutter with inverse gamma texture. EURASIP Journal on Advances in Signal Processing 2013 2013:105 